\title{
A mathematical study of the flow of nanoparticles inside periodic permeable and viscoelastic lung
}

\author{
Jyoti Kori*, Pratibha, Mohammad Junaid Abbasi \\ Department of Mathematics, Indian Institute of Technology Roorkee, Roorkee-247667, Uttarakhand, India \\ *jyotikorii@gmail.com
}

DOI 10.17586/2220-8054-2020-11-1-5-13

\begin{abstract}
Smoking and pollution are highly hazardous to human health. Most of the environmental particles are very small in size i.e. micro or nanoparticles. When these particles are inhaled, they enter from the nose and flow with the air stream into various portions of the lungs. The alveolar region, a porous media due to number of alveoli, serves as an internal biofilter medium to filter deposited particles. To analyze the behavior of this biofilter medium, we considered the periodic permeability of lungs (due to periodic breathing) together with the viscoelasticity of the lung tissues. The flow of viscous air through the porous media is modeled by using one dimensional momentum equation with Darcy's law and the velocity of particles by second law of Newton. To model the viscoelasticity, we used Kelvin-Voigt model. The finite difference method is used to solve the governing equations and MATLAB is used to solve the computational problem. The effects of various parameters, such as the Darcy number, porosity, and the breathing frequency are analyzed for flow of air, particle and viscoelasticity of lung graphically. Results show that by increasing the breathing frequency, decreasing the porosity, and decreasing the Darcy number, the viscoelastic stress increases.
\end{abstract}

Keywords: Breathing frequency, Darcy number, lungs tissue, periodic permeability, porous media, viscoelasticity.

Received: 23 October 2019

Revised: 10 December 2019

\section{Introduction}

Various literature have been presented on lungs and on its parts [1,2], few of them considered lungs as a porous media, because there were many alveoli and multiple bifurcations and some of the literature [3,4] considered lung airways as a permeable tube [5,6]. Alveoli lie at the last division of the lungs, and their work is to exchange the oxygen and carbon dioxide from air to blood and vice-versa. They are little balloon shaped air sacs and are clustered together throughout the lungs. Hazardous nanoparticles may get deposited in the alveoli and cause various lung diseases.

The toxicity of nanoparticles is right now of major concern, due to fast evolution and growth of nanotechnology [7, 8]. To date, the interactions of nanoparticles with human body are not completely understood, while an epidemiological study noted surplus incidence from fine particles derived from air pollution affects several cities of the United States [9]. When nanoparticles are merged or joined, their performance varies from larger particles. They create an extraordinarily huge risk to health, as they are assumed to be more reactive and toxic as compared to larger particles. Depending on the field of implementation, nanoparticles have various ways to enter the body, via inhalation, dermal, oral or injection exposure. Carbon nanotubes have aspect ratios which are comparable to asbestos fibers. If inhaled, then the nanotubes can probably move effortlessly through the airways down to the alveoli, where they might get stuck and can commence different diseases like asthma, COPD, emphysema, fibrosis [10,11] etc.

Biofiltration is a transpiring technology used for pollution control. It can be used for both water purification as well as air purification, with a comprehensive range of adulterants. Biofiltration uses biological means for the deterioration of pollutants in either air or water stream. It has been applied to rectify contaminated air with volatile organic compounds (VOCs) and other gases [10,12-14]. This technology is favored as a pollutant removal technique, as it has low investment and operating cost, high efficiency, reliable operating stability and low aggregate of secondary pollution [4]. Biofilters are generally porous media, i.e., a material that contains pores or voids in it. The pores are ordinarily filled with a fluid (liquid or gas) [11]. Generally, fluids flowing through the biofilter media can be exactly calculated by the accustomed models, such as the Darcy's model [7]. Darcy's law enables the analysis of laminar flow of a fluid through a porous media. The lower limit of sustainability of Darcy's law is a threshold gradient that can be required to start the flow. Darcy's law was productively used to model the flow of gas through the various mixtures. The media must have a high porosity for minimization of the pressure drop across the biofilter, quality moisture holding capacity, and a sufficiently long usage life [2]. In human lung alveolar sacs can be reviewed as a biofilter, which removes the particles from the in-breathed air. 
The objective of this study is to analyze the behavior of biofilter media (alveolar sac) with respect to Darcy number, elasticity of lungs and breathe frequency. We considered in this study the periodic permeability of lungs (due to periodic breathing) together with the viscoelasticity of the lung tissues.

\section{Mathematical modeling}

\subsection{Governing equations for viscous air flowing through airway tube}

We assumed the duct of alveolar region as a circular tube of radius $a$ as shown in Fig. 1. The motion of dusty air is along the axial direction of tube and there is no flow in radial direction. The fluid is incompressible, viscous and flow is laminar Newtonian under the time dependent pressure gradient.

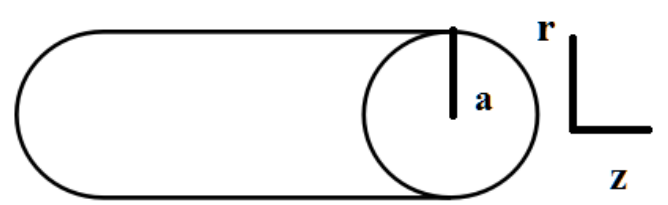

FIG. 1. Alveolar region as circular tube, where $r$ is the radial direction and $z$ is the axial direction of viscous air flow

To analyze the behavior of viscous air through the alveolar region (internal biofilter and also a porous media) Darcy law for porous media is used in one dimensional momentum equation and flow of particle is analyzed by Newton second law of motion. In our previous work [5] we focused on the effect of periodic permeability of lung. We extended our previous work by including viscoelastic property of human lung and aimed to analyze the flow behavior of air and nanoparticles inside periodically permeable human lung.

To observe the effects of lung tissue viscoelasticity, we considered human lung as a Voigt body and extended Kelvin-Voigt model by including periodic permeability of lung. So, the flow governing equations in cylindrical polar coordinate system are defined as follows $[5,15,16]$ :

$$
\begin{gathered}
\frac{\partial u}{\partial t}+\frac{u}{\epsilon} \frac{\partial u}{\partial r}=-\frac{\epsilon}{\rho} \frac{\partial p}{\partial r}+\nu\left(\frac{\partial^{2} u}{\partial r^{2}}+\frac{1}{r} \frac{\partial u}{\partial r}\right)+F \\
m \frac{\partial v}{\partial t}=k(u-v) \\
\sigma_{f}=\left(B+\frac{4 \phi}{3}\right) \frac{\partial u}{\partial r}+\left(\zeta+\frac{4 \eta}{3}\right)\left(\frac{\partial^{2} u}{\partial t \partial r}\right)
\end{gathered}
$$

where $u$ and $v$ are the velocity of air flow and dust particles respectively, $\rho$ is the air density, $\epsilon$ is the porosity, $\nu$ is the kinematic viscosity parameter, $p$ is the fluid pressure, $m$ is the mass concentration of dust particles, $k$ is the Stokes resistance coefficient, which is equal to $6 \mu a$ for spherical particles, $\mu$ being the coefficient of viscosity of the air and $a$ is the radius of the particles. $B$ and $\phi$ are bulk compression; $\zeta$ and $\eta$ are shear and bulk coefficients of viscosity. $F$ stands for the entire body force because of the existence of porous media and additional external force fields, and is formulated as:

$$
F=\left(-\frac{\epsilon \nu}{K} u+k \frac{N_{0}}{\rho}(v-u)\right)
$$

where $K$ is the periodic permeability [17], which is different from permeability. We are using periodic permeability because our breathe changes with time or we can say that our breath is periodic and the permeability of the porous medium need not to be constant. Recently, Singh et al. [19] studied buoyancy-driven free convective flow through a porous medium with periodic permeability variation. The problem becomes three-dimensional due to such a variation of permeability. The permeability of the porous medium and the free air flow velocity are assumed to be of the following forms respectively:

$$
K=\frac{K_{0}}{1-a_{0} \cos (\pi r / a)}
$$

$k_{0}$ is the mean permeability of porous medium, and $a_{0}$ is the amplitude of oscillation. 


\subsection{Initial and boundary conditions}

All the initial conditions are at $t=0$ :

$$
u=0, \quad v=0 .
$$

The boundary conditions for the fluid are same as those for classical fluid and no particle boundary conditions are required. The air velocity is set to be zero on the walls.

$$
u=0, \quad v=0, \quad \frac{\partial u}{\partial r}=0 .
$$

\subsection{Transformation of the governing equations}

In order to solve the equations numerically, we choose the physical effects which are important for us and correspondingly make the above equations dimensionless as follows:

$$
x^{*}=\frac{r}{a}, \quad P^{*}=\frac{p a^{2}}{\rho u_{0}^{2}}, \quad T^{*}=\frac{t \nu}{a^{2}}, \quad U^{*}=\frac{a u}{\nu}, \quad V^{*}=\frac{a v}{\nu}, \quad \sigma_{F}^{*}=\frac{\sigma_{f} a^{2}}{\rho \nu^{2}} .
$$

By using above quantities, we obtained the following equations ${ }^{1}$,

$$
\begin{gathered}
\frac{\partial U}{\partial T}+\frac{U}{\epsilon} \frac{\partial U}{\partial x}=-\epsilon \frac{\partial P}{\partial x}+\left(\frac{\partial^{2} U}{\partial x^{2}}+\frac{1}{x} \frac{\partial U}{\partial x}\right)+L(V-U)-\frac{\epsilon}{D a} U \\
\frac{\partial V}{\partial T}=\frac{(U-V)}{\tau},
\end{gathered}
$$

where:

$$
\begin{gathered}
D a=\frac{K}{a^{2}}, \quad L=\frac{M}{\tau}=\frac{N_{0} k a^{2}}{\mu}, \quad M=\frac{N_{0} a m}{\rho}, \quad \tau=\frac{m \nu}{a^{2} k}, \\
\sigma_{F}=\frac{\rho \nu^{3}}{a^{3}}\left[\left(B+4 \frac{\phi}{3}\right) \frac{\partial U}{\partial X}+\left(\zeta+\frac{4 \eta}{3}\right) \frac{\partial^{2} U}{\partial X^{2}}\right] .
\end{gathered}
$$

Due to smoking a person faces a breathing problem, therefore, we assumed the pressure gradient of air as a function of exponent:

$$
-\frac{\partial P}{\partial x}=P_{0} e^{-f t}
$$

where, $f$ is the breathing frequency per minute.

\section{Methodology}

The governing transformed equation is solved using finite difference method and used central difference approximation for the radial derivatives [14] and after discretization, we used MATLAB to solve computational problem. The result seemed to be converged with an accuracy on the order the time step which was chosen $\Delta t=0.00001$ and $\Delta x=0.01$ along the axial directions.

\section{Discretization of components}

For discretization of velocity $u(x, T)$ or $\mathrm{u}\left(x_{i}, T_{j}\right)$ or $u(i, j)$, we used following step sizes:

$$
x_{i}=i \Delta x,(i=0,1,2,3, \ldots, N),
$$

where

$$
x_{N}=1.0, \quad T_{j}=(j-1) \Delta t, \quad(j=1,2, \ldots) .
$$

Additionally, at $x=0$, following approximation is applicable in equation (8), When $x \rightarrow 0$, we found that the denominator is also approaching to zero and make the term indeterminate, which is inappropriate for the physical state of the respiratory system. Therefore, to remove this kind of inconsistency we used L'Hôpitals rule of limit here:

$$
\lim _{x \rightarrow 0} \frac{1}{x} \frac{\partial U}{\partial x}=\lim _{x \rightarrow 0} \frac{\partial^{2} U}{\partial x^{2}}
$$

\footnotetext{
${ }^{1}$ For simplicity we drop the asterisk in all the dimensionless equations.
} 
so, the equation 8 at $x=0$ becomes:

$$
\begin{aligned}
U_{i, j+1}=\left(1-L \Delta t-\left(\frac{\epsilon}{D a}\right) \Delta t-4 r_{1}\right) U_{i, j}+ & \left(2 r_{1}-\frac{U_{i, j} r_{1} \Delta x}{2 \epsilon}\right) U_{i+1, j}+ \\
& \left(2 r_{1}+\frac{U_{i, j} r_{1} \Delta x}{2 \epsilon}\right) U_{i-1, j}+L \Delta t V_{i, j}+P_{0} e^{-f j \Delta t} \epsilon \Delta t,
\end{aligned}
$$

and at $0<x \leqslant 1$ :

$$
\begin{aligned}
U_{i, j+1}=\left(1-L \Delta t-\left(\frac{\epsilon}{D a}\right) \Delta t-2 r_{1}\right) U_{i, j} & +\left(r_{1}-r_{1} \Delta x \frac{U_{i, j}}{2 \epsilon}+\frac{r_{1}}{2 i}\right) U_{i+1, j}+ \\
& \left(r_{1}+\frac{r_{1} \Delta x U_{i, j}}{2 \epsilon}-\frac{r_{1}}{2 i}\right) U_{i-1, j}+L \Delta t V_{i, j}+P_{0} e^{-f j \Delta t} \epsilon \Delta t
\end{aligned}
$$

where $\left(\frac{\Delta t}{\Delta x^{2}}\right)=r_{1}$.

The equation (9) transforms as:

$$
\begin{gathered}
V_{i, j+1}=\left(1-\frac{\Delta t}{\tau}\right) V_{i, j}+\frac{\Delta t}{\tau} U_{i, j}, \\
\left(\sigma_{F}\right)_{i, j}=\frac{\rho \nu^{3}}{a^{3}}\left[\left(B+4 \frac{\phi}{3}\right)-\left(\left(\zeta+\frac{4 \eta}{3}\right) \frac{1}{\Delta t}\right) \frac{U_{i+1, j}}{2 \Delta r}\right]+ \\
\frac{\rho \nu^{3}}{a^{3}}\left[\left(\left(\zeta+\frac{4 \eta}{3}\right) \frac{1}{\Delta t}-\left(B+4 \frac{\phi}{3}\right)\right) \frac{U_{i+1, j}}{2 \Delta r}+\left(\zeta+\frac{4 \mu}{3}\right)\left(\frac{u_{i+1, j+1}-u_{i-1, j+1}}{2 \Delta t \Delta r}\right)\right] .
\end{gathered}
$$

The initial and boundary conditions in discretized form are as follows:

$$
U_{i, 1}=V_{i, 1}=0, \quad U_{2, j}=U_{0, j} ; \quad U_{N+1, j}=0 .
$$

\section{Results and Discussion}

Numerical computations have been carried out using the following parameter values $[1,9,18]$ :

$$
\begin{aligned}
& P_{0}=101.325 \mathrm{kPa}, \quad m=0.0002 \mathrm{~kg} / \mathrm{l}, \quad d_{p}=100 \mathrm{~nm}, \quad \rho=1.185 \mathrm{~kg} / \mathrm{m}^{3}, \quad \nu=1.52 \mathrm{~s}^{-1}, \\
& N_{0}=0.02504 \cdot 10^{12} / \mathrm{m}^{3}, \quad a=0.5 \mu \mathrm{m}, \quad \epsilon=0.6, \quad B=3000 \mathrm{~kg} /\left(\mathrm{m} \mathrm{sec}^{2}\right), \quad \phi=400 \mathrm{~kg} /(\mathrm{m} \mathrm{sec}), \\
& \zeta=10 \mathrm{~kg} /(\mathrm{m} \mathrm{sec}), \quad f=0.2 \text { to } 0.3 \text { per sec (for diseased, } 0.2 \text { to } 0.5 \text { per sec). }
\end{aligned}
$$

Velocities of air and dust particles on the wall have been represented graphically with variable axial positions, time, and porosity and Darcy number.

\subsection{Effect of porosity on internal biofilter}

We defined void ratio as the ratio of free space volume in the biofilter to the total volume of biofilter. There are voids in a biofilter which are not filled by any medium. If the void ratios are high then it reduces the clogging and allow the air to move freely in biofilter.

From Fig. 2(a) it is clear that at porosity $\epsilon=0.6$, and Darcy number $=0.01$ the velocity of the air increases with time. It has attained maximum velocity at the center of the tube at $x=0$ and occupied maximum value earlier at $t=0.1$. After that, the velocity of air decreases simultaneously as time increases and became zero on the tube wall. Due to classical flow, initially the effects of particles are almost negligible, therefore the highest velocity is attained. Later the particle hinders the flow and the subsequent velocity reduces:

In Fig. 2(b) at porosity $\epsilon=0.9$, the velocity of the air increases with time. It obtains maximum velocity at $t=0.2$ at the center of the tube and after that decreases as time increases. The velocity depends on the porosity. From Figs. 2(a) and 2(b) it is quite clear that at porosity $\epsilon=0.9$, the velocity profiles for the air flow are significantly more than that at porosity $\epsilon=0.6$. Figs. 3(a) and 3(b) show the velocities for the dust particles near the starting point of the tube with the time at porosity $(\epsilon) 0.6$ and 0.9 respectively. Velocities of the dust particles increase when radial distance increases and increasing values of time. From the figures, we can see that the radial parameter works gradually to exhibit its effect, i.e. velocity of the dust particles increases frequently with time. From Figs. 3(a) and 3(b) it is clear that at porosity $\epsilon=0.9$, the velocity profiles for the dust particles increase more than that at porosity $\epsilon=0.6$. 


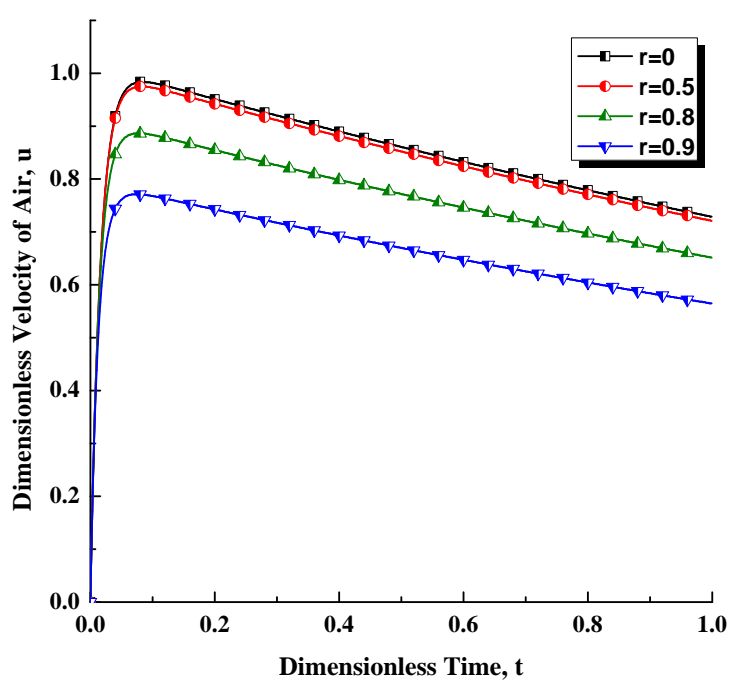

(a)

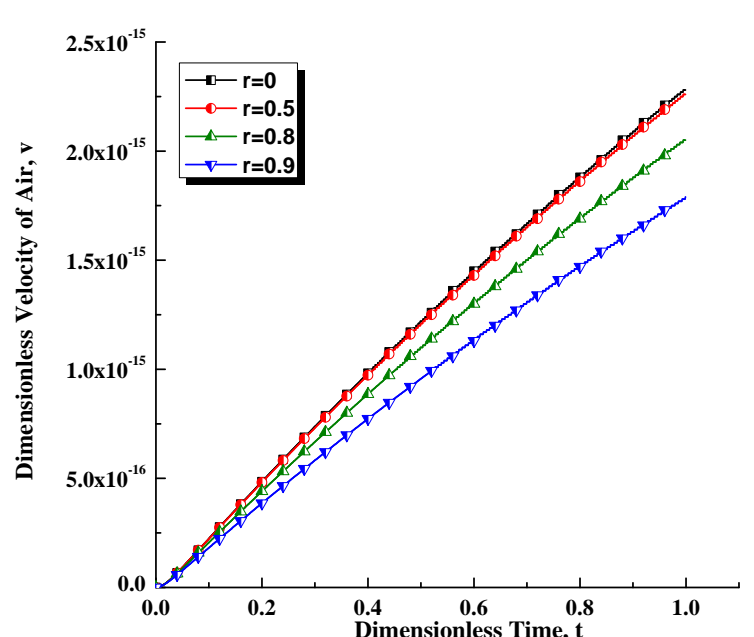

(b)

FIG. 2. Velocity profile at $K_{0}=0.1, d=100 \mathrm{~nm}, \epsilon=0.6$, and $f=0.2$ per sec for different radial positions of (a) air and (b) particle flows

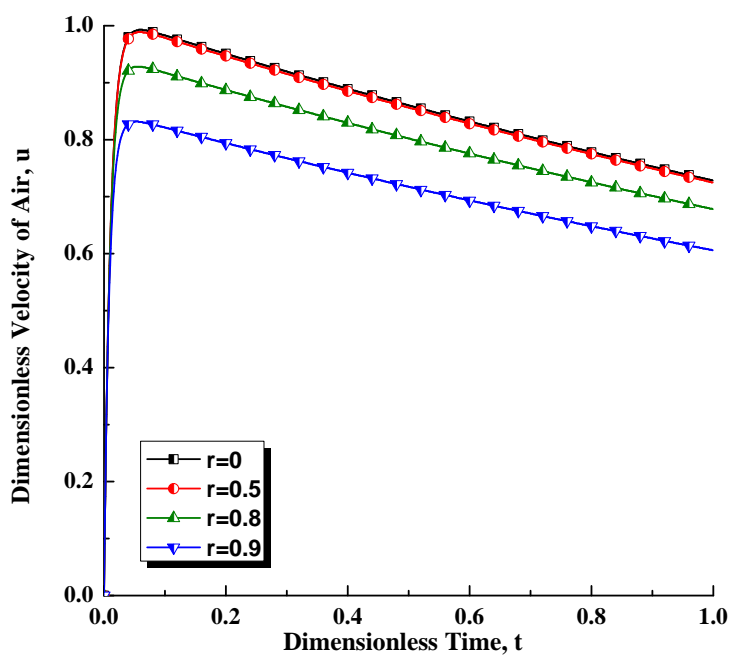

(a)

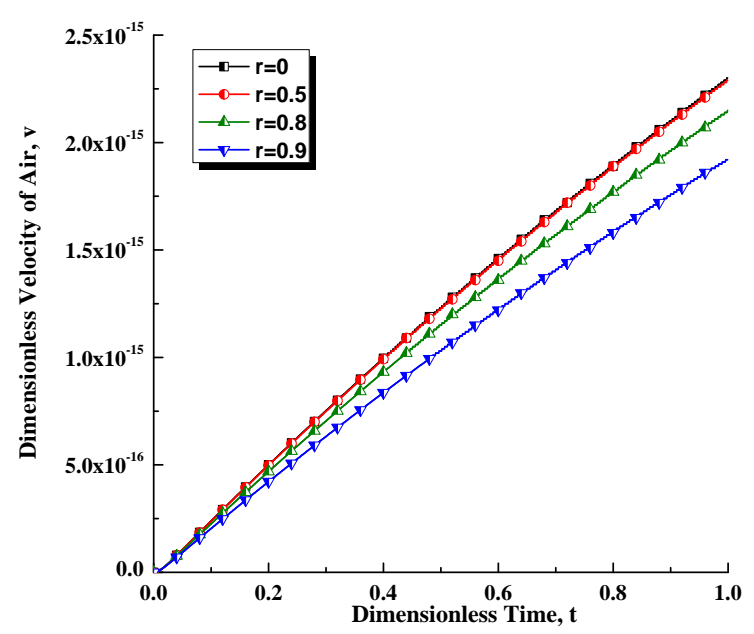

(b)

FIG. 3. Velocity profiles at $K_{0}=0.1, d=100 \mathrm{~nm}, \epsilon=0.9$, and $f=0.2$ per sec for different radial positions of (a) air and (b) particle flows

\subsection{Effect of Darcy number on internal biofilter}

Figures 4 and 5 are shown the variations of velocity for the fluid for different values of radial positions ( $x$ with time. As $x$ increases, the velocity decreases for the fluid. Velocity of the fluid at Darcy number $10^{-1}$ is greater than at Darcy number $10^{-2}$ for fixed axial position: From Figs. 4(a) and 4(b), it is clear that the velocity of the air increases regularly as value of time increases. As well as we found from Figs. 5(a) and 5(b) velocity of particle at Darcy number $10^{-1}$ is greater than at Darcy number $10^{-2}$ for fixed axial position. 


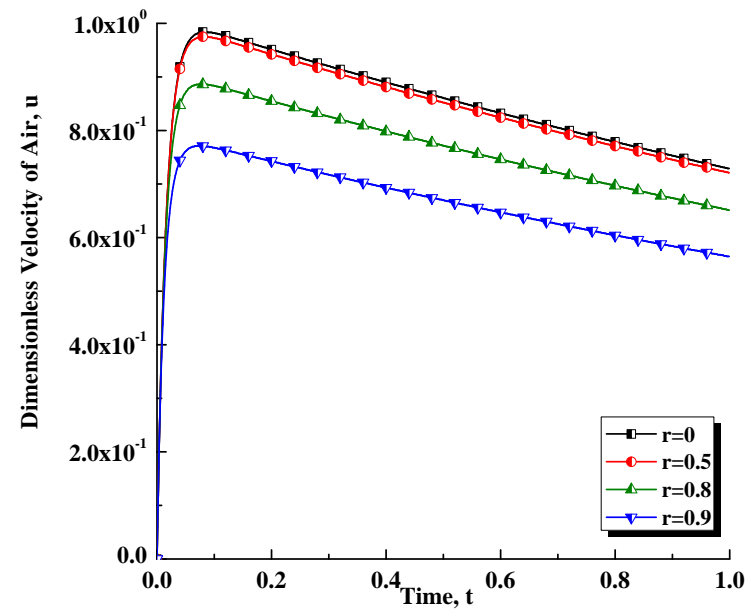

(a)

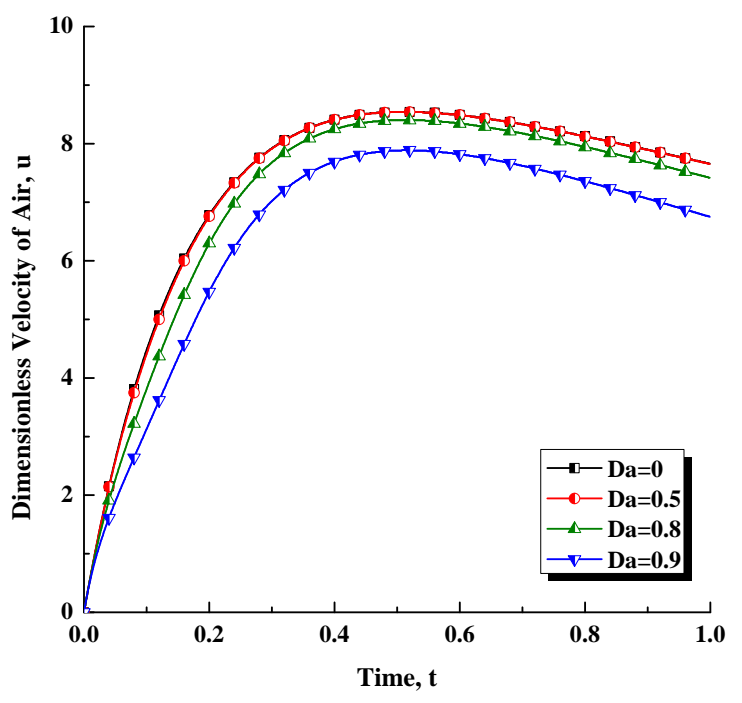

(b)

FIG. 4. Velocity profiles for different radial positions of air for (a) $D a=10^{-2}$, and (b) $D a=10^{-1}$ at $K_{0}=0.1, d=100 \mathrm{~nm}, \epsilon=0.6$, and $f=0.3$ per sec.



(a)

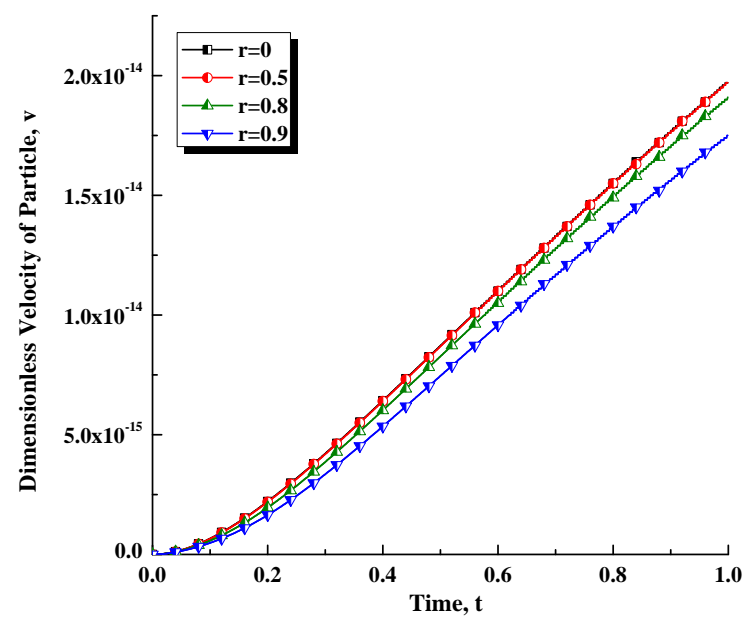

(b)

FIG. 5. Velocity profiles for different radial positions of particle for (a) $D a=10^{-2}$, and (b) $D a=$ $10^{-1}$ at $K_{0}=0.1, d=100 \mathrm{~nm}, \epsilon=0.6$, and $f=0.3$ per sec.

\subsection{Effect of breathing frequency on viscoelastic stress}

Figure 6 shows how increasing value of breathing frequency increases the viscoelastic stress, when we take Darcy number as 0.01 , porosity 0.9 and varies the breathing frequency from $f=0.2$ to 0.5 per sec. At $f=0.2$, value of viscoelastic stress is very low and by increasing value of $f$ from 0.2 to 0.5 we found increment in the viscoelastic stress. Due to an increase in breathing frequency, which can be caused by any lung disease such as COPD or asthma, the velocity of air as well as particles increases. Due to increment in the fluid velocity the pressure in alveolar section increases, which causes increment in the viscoelastic stress. 


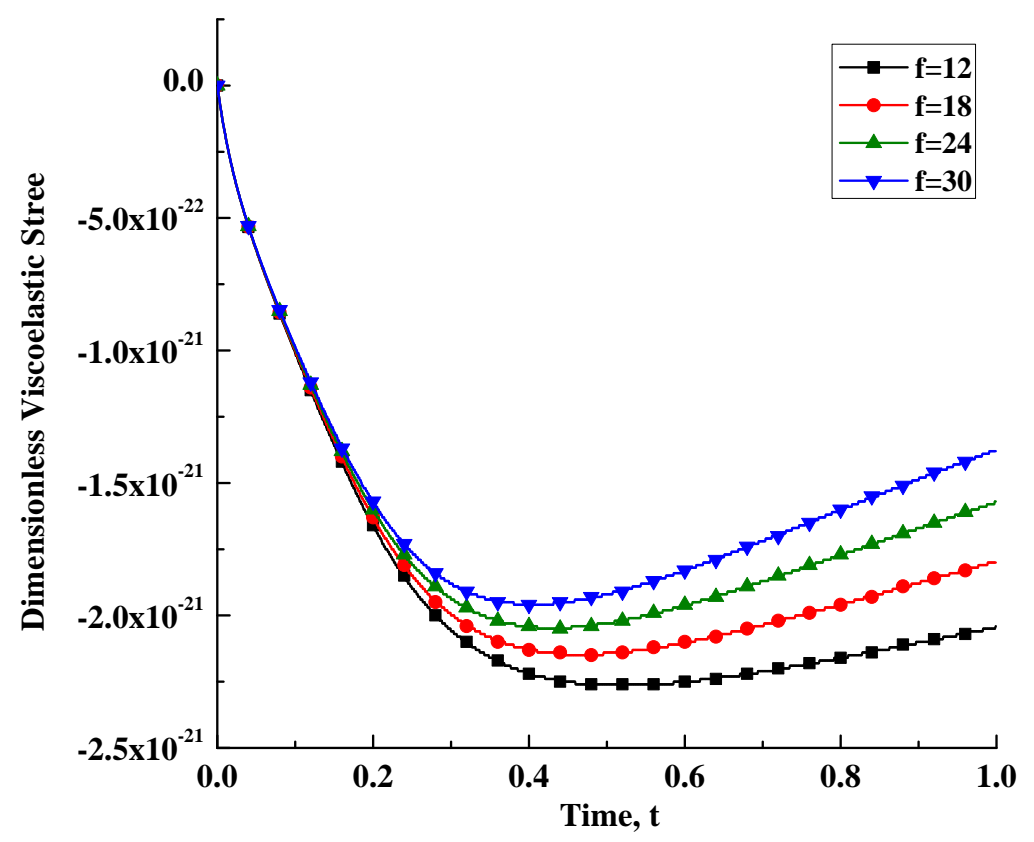

FIG. 6. Effect of breathing frequencies from $0.2-0.5$ per sec on viscoelastic stress at $D a=10^{-2}$, $K_{0}=0.1, d=100 \mathrm{~nm}$, and $\epsilon=0.6$.

\subsection{Effect of porosity on viscoelastic stress}

In Fig. 7, we have plotted viscoelastic stress with different values of porosity ( $0.6 \& 0.9)$ at Darcy number 0.01 and breathing frequency at 0.26 per sec.

From Fig. 7, we found by increasing porosity from 0.6 to 0.9 , the viscoelastic stress first decreases then increases. By increasing the porosity from 0.6 to 0.9 , the number of alveoli increases which allow flow of air very freely and caused reduction in the stress of lung tissue in some extent.

\subsection{Effect of Darcy number on viscoelastic stress}

Darcy number is calculated using permeability of medium which depends on porosity of medium, so it is safe to say that Darcy number depends on porosity of medium. So, if Darcy number increases media becomes more fluidic. We have plotted the stress vs Darcy number varying from 0.1 to 0.001 with keeping breathing frequency at 0.26 per sec and porosity at 0.6. From Fig. 8, we can see that as the Darcy number increases the value of viscoelastic stress decreases. The value of stress at Darcy number 0.001 is not quite zero but it has comparatively very small value. The minimum value is obtained for Darcy number 0.1 at $x=0.5$. From that, we can see that viscoelastic stress decreases as we increases value of Darcy number, which make the flow more fluidic.

\section{Conclusion}

In this study, to analyze the behavior of biofilter media, we considered the periodic permeability of lungs (due to periodic breathing) together with the viscoelasticity of the lung tissues. Mathematical modeling is used to model the problem and MATLAB is used to solve the problem computationally. The effects of various parameters, such as Darcy number, porosity, and breathing frequency are calculated on flow of air and particles. Additionally, effects of breathing frequency, porosity, Darcy number are found on viscoelastic stress for diseased lung (such as COPD, Asthma). After performing the numerical computation we found that by increasing the media porosity velocity of air and particle increases. By increasing the Darcy number, the velocity of air and particle increases. By increasing the breathing frequency, decreasing the porosity and decreasing the Darcy number viscoelastic stress increases. Hence, we can conclude from this study that Darcy number, elasticity of lungs and breathing frequency affect the flow of air and particles inside internal biofilter media (alveolar sac) of lung. 


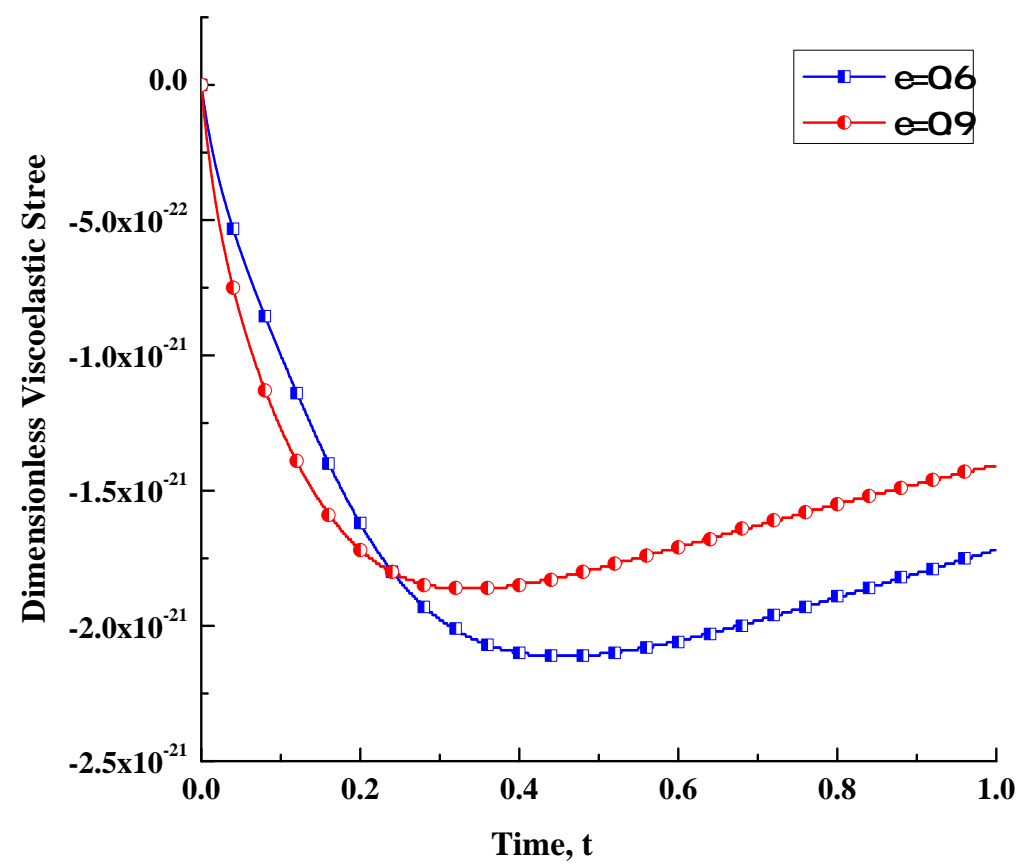

FIG. 7. Effect of porosity $(\epsilon=0.6$ and 0.9$)$ of biofilter media on viscoelastic stress at $D a=10^{-2}$, $K_{0}=0.1, d=100 \mathrm{~nm}$, and $f=0.26$ per sec.

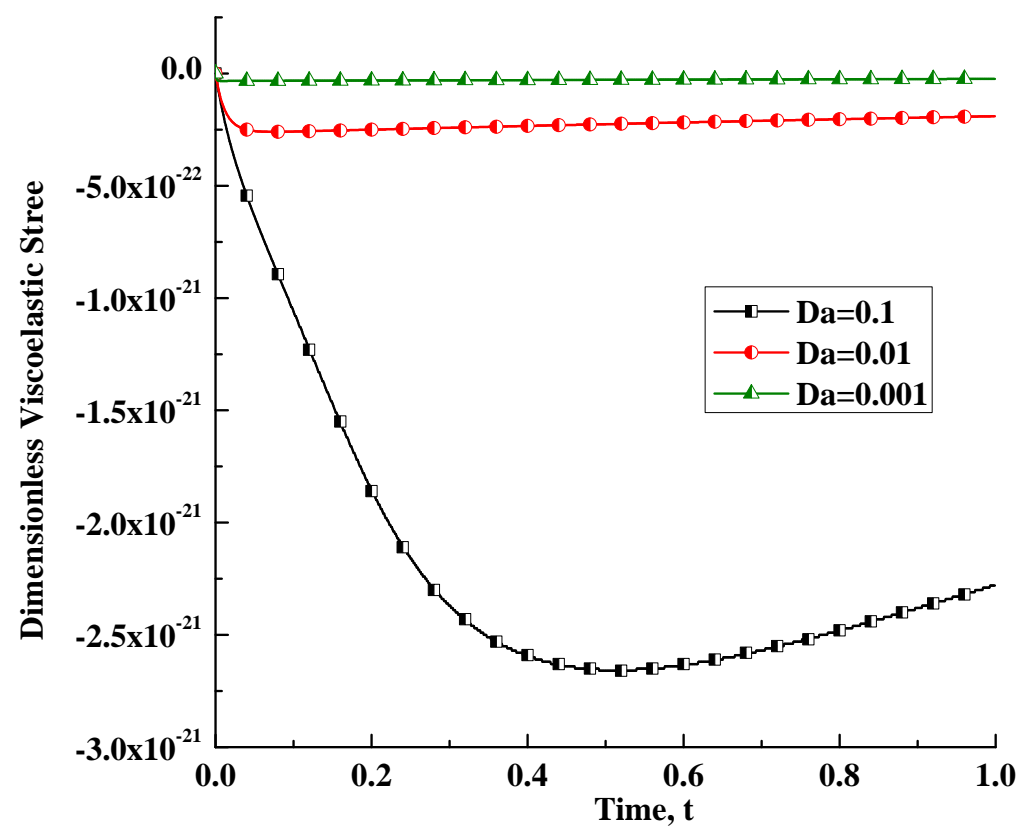

FIG. 8. Effect of Darcy number for 0.1 and 0.01 on viscoelastic stress at $\epsilon=0.6, K_{0}=0.1$, $d=100 \mathrm{~nm}$, and $f=0.26$ per sec.

\section{Acknowledgements}

One of the authors, Jyoti Kori, is thankful to Ministry of Human Resource Development India (Grant Code: MHR-02-23-200-44) for providing fund and support while writing this manuscript. 


\section{Conflict of Interest}

No conflict of interest.

\section{References}

[1] Cengel Y., Cimbala J. Solutions manual for fluid mechanics: Fundamentals and applications. Bukupedia, McGraw-Hill Companies, Incorporation, New York, 2006.

[2] Kafle G., Chen L., et al. Field evaluation of wood bark-based down-flow biofilters for mitigation of odor, ammonia, and hydrogen sulfide emissions from confined swine nursery barns. Journal of environmental management, 2015, 147, P. 164-174.

[3] Wei Z., Liu X., et al. Gas dimethyl sulfide removal in biotrickling filtration. Open Access Scientific Reports, 2013, 2 (702).

[4] Zhang Y., Finlay W.H. Measurement of the effect of cartilaginous rings on particle deposition in a proximal lung bifurcation model. Aerosol Science and Technology, 2005, 39, P. 394-399.

[5] Kori J., Pratibha. Effect of periodic permeability of lung airways on the flow dynamics of viscous fluid. Nanosystems: Physics, Chemistry, Mathematics, 2019, 10 (3), P. 235-242.

[6] Kori J., Pratibha. Effect of Periodic Permeability of Lung Tissue on Fluid, Velocity and Nonspherical Nanoparticle Filtration. International Journal of Applied and Computational Mathematics, 2019, 5 (49), P. 1-15.

[7] Darcy H. Les fontaines publiques de la ville de Dijon. Dijon, Vector Dalmont, Paris, 1856.

[8] Nel A., Xia T., et al. Toxic potential of materials at the nanolevel. Science, 2006, 311 (5761), P. $622-627$.

[9] Hoet P., Hohlfeld I., Salata O. Nanoparticles-known and unknown health risks. Journal of Nanobiotechnology, 2004, 2 (12), P. 1-15.

[10] Sharma P. Free convection effects on the low past a porous medium bounded by a vertical infinite surface with constant saction and constant heat flux. Journal of physics D-Applied physics, 1992, 25, P. 162-166.

[11] Siddiqui A.M., Siddiqa S., Naqvi A.S. Effect of constant wall permeability and porous media on the creeping flow through round vessel. Journal of Applied and Computational Mathematics, 2018, 7 (2), P. 1-6.

[12] Anet B., Lemasle M., et al. Characterization of gaseous odorous emissions from a rendering plant by gc/ms and treatment by biofiltration. Jounral of Environmental Management, 2013, 128, P. 981-987.

[13] Malhautier L., Cariou S., et al. Treatment of complex gaseous emissions emitted by a rendering facility using a semi-industrial biofilter. Journal of Chemical Technology and Biotechnology, 2014, 11 (21), P. 426-430.

[14] Smith G. Numerical Solution of Partial Differential Equations, Oxford University Press, New York, 1985.

[15] Nithiarasu P., Ravindran K. A new semi-implicit time stepping procedure for buoyancy driven flow in a fluid saturated porous medium. Computer methods in applied mechanics and engineering, 1998, 165 (1), P. 147-154.

[16] Saini A., Katiyar V.K., Pratibha. Numerical simulation of gas flow through a biofilter in lung tissue. World Journal of Modelling and Simulation, 2015, 11 (1), P. 33-42.

[17] Meyers, Chawla. Section 13.10 of Mechanical Behaviors of Materials, Mechanical behavior of Materials, Cambridge University Press, 1999, P. 570-580. Prentice Hall, Inc.

[18] Flora J., Hargis R., et al. The role of pressure drop and flow redistribution on modeling mercury control using sorbent injection in baghouse filters. Journal of the Air \& Waste Management Assocation, 2006, 56 (3), P. 343-349.

[19] Singh P., Mishra J.K., Narayan K.A. Free convection along a vertical wall in a porous medium with periodic permeability variation. Int. J. Numer. Anal. Methods Geomech., 1989, 13, P. 443-450. 